\title{
Heart Rate Variability Estimation with Joint Accelerometer and Gyroscope Sensing
}

\author{
Olli Lahdenoja ${ }^{1}$, Tero Hurnanen ${ }^{1}$, Mojtaba Jafari Tadi ${ }^{1,2}$, Mikko Pänkäälä ${ }^{1}$, Tero Koivisto ${ }^{1}$ \\ ${ }^{1}$ Technology Research Center, University of Turku, Finland \\ ${ }^{2}$ Faculty of Medicine, University of Turku, Finland
}

\begin{abstract}
This paper describes a method for estimation of heart rate (HR) and heart rate variability $(H R V)$ with accelerometers and gyroscopes. We denote this joint seismocardiography (SCG) and gyrocardiography (GCG) approach as $S C G / G C G$. In principle, SCG which is a well known method measures the linear mechanical movements of the heart and GCG is a new technique which measures angular motion due to the chest micro-vibrations caused by myocardial rotation. As electrocardiography (ECG), they can also be performed in non-invasive manner using a device in contact to subjects skin, for example. Our method extracts HRV parameters based on single-axis and multi-axes autocorrelation analysis (1-AC and 6-AC) of all simultaneously captured $S C G / G C G$ axes. The results of each axes are combined to maintain reliable $H R$ - and $H R V$. We validate our results with a comparison study between simultaneous ECG and SCG/GCG recordings using a study group of 29 healthy male volunteers. The study provides a promising approach for HRV estimation with modern wearable devices.
\end{abstract}

\section{Introduction}

In this paper, we consider accurate extraction of heartbeat intervals with modern micro electromechanical (MEMS) accelerometers and gyroscopes. Seismocardiography (SCG) is a well known non-invasive method for extracting information of heart mechanical movements [1]. A tri-axial MEMS accelerometer can be used to obtain a 3axes SCG waveform. This can be complemented by using a tri-axial gyroscope sensor (preferably MEMS). The gyroscope measures the angular velocities of the chest as a response to the motion of the heart, thus we use the term Gyrocardiography (GCG) [2]. The joint usage of seismo- and gyrocardiography is denoted in this paper as SCG/GCG, where both sensor signals are acquired from miniature 3axis MEMS sensors attached on the sternum of the patient in a non-invasive manner .
A recent review on SCG was given in [3]. The capability of SCG and ballistocardiography (BCG) - the recording of the reactionary forces of the body invented by Gordon in 1877 [4] - for estimating HRV has been previously reported by $[3,5,6]$. HRV reflect a variety of aspects of human heart and autonomous nervous system. For instance, emotions are related to HRV and the operation of human autonomous nervous system [7]. HRV is also affected by certain drugs, transient coronary occlusion, and exercise which may result in prolonged cardiac cycle intervals, while the breathing related heart rate modulation of a human is called respiratory sinus arrhythmia. Usually, a reduced HRV relates to many unwanted heart conditions, for example, mortality risk after acute myocardial infarction is higher for patients with low HRV [8].

Here we propose that the simultaneous usage of gyroscope sensor can supplement the measured SCG by attaining information on small-scale body vibrations induced by the pumping of the heart muscles $[9,10]$. While being a non-invasive method and possessing high practical clinical value, ECG devices have not yet been widely accepted for very long term remote screening of the patients, since the attached electrodes may irritate the skin of the patient (excluding implantable devices). The novelty introduced in this paper is that we combine the three SCG axes and three GCG axes to obtain more reliable HRV parameters (see Fig. 1). Thus, long term monitoring of the heart with this kind of method could be possible in the future.

\section{Autocorrelation Technique}

\subsection{Single-axis autocorrelation}

A challenge in both SCG and BCG with regard to interbeat estimation is that other motions such as breathing (and other muscles) disturb the acquisition of the heart signal. Also, the signal is not typically as clear as in ECG, where the QRS complex (R-R interval) is usually rather easy to segment from the other parts of the signal. Thus, it is usually required that as a prerequisite to the signal acquisition the subject needs to be still. Although ECG can suffer 

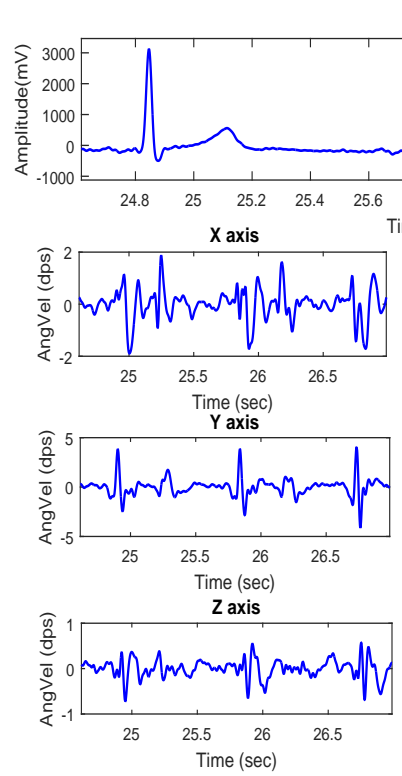

ECG
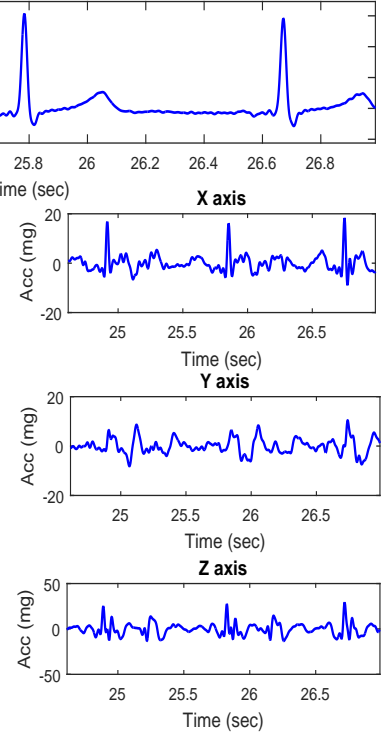

Figure 1. ECG, 3-axis accelerometer (right insets) and gyroscope axes (left insets). Accelerometer $\mathrm{Z}$ axis is used in the case of 1-AC (autocorrelation) and all axes in the case of 6-AC.

from motion artifacts, the situation is much more severe in the case of BCG and SCG. Even if the subject is still, the waveforms of the SCG and BCG signals are typically more complex and require different means for extracting HR and HRV than in ECG.

Single axis autocorrelation (denoted in this paper as 1AC method) has been previously applied to ECG and BCG analysis (see [11] [12]). The 1-AC for discrete signal is defined as

$$
\phi(\sigma)=1 / N * \sum_{n=0}^{N-1} x(n) x(n+\sigma),
$$

where $\phi(\sigma)$ is the autocorrelation function of length $N$ segment taken from the signal [13] and $\sigma$ is the lag for discrete signal. $\phi(\sigma)$ has a large value when the signal $x(n)$ is similar with $x(n+\sigma)$. For periodical $x(n)$ the AC function $\phi(\sigma)$ has peaks in its response [12] [13]. We use the accelerometer $\mathrm{Z}$ axis, which is the same axis as gravity when the subject is in supine position when applying the 1-AC method throughout this paper. We also extend the single axis autocorrelation to multiple-axis autocorrelation (6-AC), where all available accelerometer and gyroscope axes are applied.

In our case $N$ in Equation 1 is determined by the sampling rate $F_{s}$ of the sensor and the used window size $T_{w k}$ in seconds, here we use $F s=800 \mathrm{~Hz}$ and window size 2.5 s, i.e. $N=2000$ samples for both the signal $x(n)$ and the shifted signal. The shifted signal is extended to overlap between all existing values of the signal $x(n)$ itself. For single axis, bandpass filtered version of the original signal is first acquired, and it is sampled at constant $T_{i n t}=1$ second intervals. The overlap between two windows becomes $1.5 \mathrm{~s}$ (assuming $2.5 \mathrm{~s}$ full autocorrelation). Fig. 2 shows the autocorrelation results for a generated signal with and without windowing. Observe that in the bottom insets the first peak is actually the autocorrelation of the generated signal with itself, and detecting it would not result in useful information (see Fig. 2).

In principle, contraction of the left ventricle (systole) yields aortic valve opening (AO) and results in the first heart sound, S1, while by the beginning of ventricular relaxation (diastole) and after closure of aortic valve (AC) the second heart sound, S2 occurs [6]. Before applying the 1-AC method, the signal of the single axis is first multiplied by a triangular waveform, which emphasizes the leftmost values within the sliding autocorrelation window. This in itself is not sufficient, since it is possible that instead of finding the peak corresponding to two adjacent true beats, the autocorrelation can find the $\mathrm{AO}-\mathrm{AC}$ interval within single beat. This is because the systolic and diastolic peaks both are with significant signal power in contrast to ECG, where the R peaks are typically dominant. Therefore, the leftmost part ( $\phi<\phi_{0}, \phi_{0}=3 / 5$ seconds) of the resulting 1-AC window is assigned to zero. After that, the dominant peak of the 1-AC window is assumed to contain the dominant instant heart rate component.
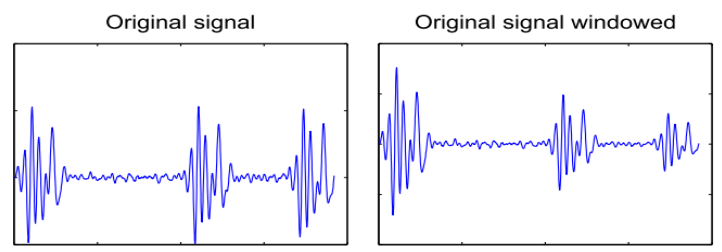

Autocorrelation of original signal Autocorrelation of windowed signal
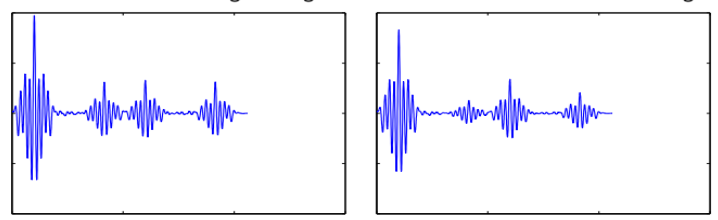

Figure 2. The figure shows first the original signal, and then the original signal windowed with a steadily decreasing windowing function (upper right corner). The autocorrelation of the original signal (lower left corner) shows that the first peak from the left (corresponding to signal itself) is the largest, but the other peaks are difficult to distinguish. The last image (lower right corner) shows that the windowing function allows for separating the peaks after the leftmost part of the AC signal has been thresholded to zero. 


\subsection{From Single-axis to Multi-axes Auto- correlation}

The multi-axes case autocorrelation for accelerometer and gyroscope is defined as $\phi(\sigma)_{k}$, where where axes $k$ are $1=A C C_{x}, 2=A C C_{y}, 3=A C C_{z}, 4=G Y R O_{x}$, $5=G Y R O_{y}, 6=G Y R O_{z}$ for the SCG signal components $A C C$ and GCG signal components $G Y R O$. The 1-AC function is applied for each axes simultaneously and the RR interval time series are extracted. Then, if the RR interval is the first acquired RR interval the selected output of the combined axes is chosen as the median or mean of these six values (initialization). After that, one of the six interval of each axis closest to the previously determined combined interval is chosen as the output (update).

In certain conditions where a weak signal power peak is located in between two higher amplitude peaks, within the same 1-AC window, the algorithm may misinterpret an unnecessary long RR interval. Then, the algorithm prefers the higher amplitude peak pair and select the longer interval instead of the correct (previous) one. Furthermore, in worst case the following interval estimations could produce the same problem, if at least one of the six axes would indicate to a too long false interval. These issues could be avoided by more advanced (and computationally complex) axes combining techniques.

\section{Experimental Configuration}

\subsection{Test Set-up and Data Collection}

We used a $(3 \mathrm{~mm} \times 3 \mathrm{~mm} \times 1 \mathrm{~mm})$ triple-axis, lowpower, capacitive digital accelerometer (Freescale Semiconductor, MMA8451Q, Austin, TX, USA) and an (3 mm $\times 3 \mathrm{~mm} \times 0.9 \mathrm{~mm}$ ) ultra-accurate, low power, low noise, 3-axis MEMS angular rate sensor (Maxim Integrated, MAX21000, San Jose, CA, U.S.). The sensors were attached to the chest of the test subjects with double sided tape without hair removal from the chest. We also captured simultaneous ECG (Texas Instruments ADS1293) as a reference. The SCG/GCG and ECG data were captured simultaneously to a memory stick and were processed offline.

We collected SCG/GCG data and ECG reference from 29 individuals, which were advised to lie in supine position during the acquisition. The demographic of the subjects were as follows (min-max, mean, standard deviation): age (23-41, 29.15, 4.73 years), height (170-190, 178.48, $5.91 \mathrm{~cm})$, weight $(60-98,76.32,11.20 \mathrm{~kg})$ and BMI $(17.53-$ $\left.29.4,23.92,3.00 \mathrm{~kg} / \mathrm{m}^{2}\right)$. We extracted both time and frequency domain parameters, which were calculated using Kubios software [14] from the raw interbeat interval data obtained in Matlab. For ECG we used well known PanTompkins approach [15] for generating the ground truth.

\subsection{The Extracted HRV Indices from the Kubios software}

We report the results of 1-AC method in comparison with 6-AC method so that the ECG is used as a reference. The time domain parameters considered here are mean RR intervals, which are calculated for each recording separately (both 1-AC and 6-AC against ECG), standard deviation (STD) of RR intervals (SDNN), mean heart rate (HR), STD HR, and RMSSD (Square root of the mean squared differences between successive RR intervals). Additionally NN50 (number of successive RR intervals differing more than 50ms), pNN50 (probability of NN50 against total number of RR intervals), HRV triangular index (derived from the RR interval histogram) and TINN which is the width of the RR interval histogram are reported.

The frequency domain parameters considered here are the FFT (Fast Fourier Transform) and AR (Autoregressive model) spectrum based features. More specifically, frequency bands such as very low Frequency (VLF), low frequency (LF), and high frequency (HF) peak frequencies and power ratio LF/HF [14] are being reported.

\section{Results and Discussion}

\subsection{Time-domain HRV results}

Table I shows the mean error of 1-AC and 6-AC methods against ECG. It can be observed, that only in the cases of NN50 and pNN50 the 6-AC method's performance is decreased against the 1-AC method. As NN50 is defined as the number of successive pairwise RR intervals differing more than $50 \mathrm{~ms}$, it seems that the $6-\mathrm{AC}$ method filters out some of these, due to its characteristics as it tends to choose the next RR among the 6 available axis, so that the difference between to the previous RR interval is minimized. Thus, although the 6-AC method provides robustness to beat interval estimation as the SCG/GCG signals are more difficult to analyze than ECG, it should be taken into account that (p)NN50 like parameters performance might suffer from the applied technique. However, the most important HRV parameters Mean RR and STD RR (as well as HR and STD HR) and RMSSD are improved clearly through the use of 6-AC method. Observe, that the lengths of the recordings varied between that $1 \mathrm{~min} 44 \mathrm{~s}$ and $26 \mathrm{~min} 45 \mathrm{~s}$.

\subsection{Frequency-domain results}

Table II shows the mean error between 1-AC and 6-AC methods with respect to ECG using the frequency domain parameters extracted using the Kubios software. The frequency domain parameters showed improvement in all of the selected cases. 


\begin{tabular}{|c||c|c|}
\hline Mean error vs. ECG & 1-AC & 6-AC \\
\hline \hline Mean RR (ms) & 3.3608 & 2.2278 \\
\hline STD RR (ms) & 7.0882 & 2.5936 \\
\hline Mean HR (1/min) & 0.2162 & 0.1565 \\
\hline STD HR (1/min) & 0.6335 & 0.2178 \\
\hline RMSSD (ms) & 11.8449 & 5.2855 \\
\hline NN50 (count) & 7.9310 & 15.1034 \\
\hline pNN50 (\%) & 2.5576 & 4.5384 \\
\hline RR tri-index & 3.1595 & 2.5587 \\
\hline TINN (ms) & 46.5813 & 27.9606 \\
\hline
\end{tabular}

Table 1. Time domain HRV parameters.

\begin{tabular}{|c||c|c|}
\hline Mean error vs. ECG & 1-AC & 6-AC \\
\hline \hline FFT VLF (peak) $(\mathrm{Hz})$ & 0.0019 & 0.0003 \\
\hline FFT LF (peak) $(\mathrm{Hz})$ & 0.0121 & 0.0075 \\
\hline FFT HF (peak) (Hz) & 0.0171 & 0.0115 \\
\hline FFT LF/HF (power ratio) & 0.2692 & 0.2305 \\
\hline AR VLF (peak) (Hz) & 0.0012 & 0.0000 \\
\hline AR LF (peak) (Hz) & 0.0098 & 0.0027 \\
\hline AR HF (peak) (Hz) & 0.0193 & 0.0132 \\
\hline AR LF/HF (power ratio) & 0.2401 & 0.2169 \\
\hline
\end{tabular}

Table 2. Frequency domain HRV parameters.

\section{Conclusion}

We presented a novel approach for estimating HRV using 3-axis accelerometer and 3-axis gyroscope so that the axes are combined to obtain a more reliable HRV estimate. The presented approach could be embedded into modern wearable/implantable devices equipped with miniature MEMS sensors. Future work can include extending the study for a larger number of subjects, and studying possibilities for HRV estimation in other locations of the MEMS sensor (than on the chest only). Furthermore, in this paper the subject under test was asked to maintain in still position, and one interesting direction for future research would be to study possibilities of applying the HRV analysis while the subject is moving.

\section{References}

[1] Bozhenko BS. Seismocardiography- a new method in the study of functional conditions of the heart. Terapevticheskii Arkhiv 1961;33:55-64.

[2] Jafari Tadi M, Lehtonen E, Pänkäälä M, Saraste A, Vasankari T, Teräs M, Koivisto T. Gyrocardiography: a new non-invasive approach in the study of mechanical motions of the heart. concept, method and initial observations. In Engineering in Medicine and Biology Society, EMBC, 2016 Annual International Conference of the IEEE. Aug 2016; 1-4.

[3] Inan O, Migeotte PF, Park KS, Etemadi M, Tavakolian K, Casanella R, Zanetti J, Tank J, Funtova I, Prisk G, Di Rienzo M. Ballistocardiography and seismocardiography: A review of recent advances. Biomedical and Health Informatics IEEE Journal of July 2015;19(4):1414-1427. ISSN 2168-2194.
[4] Gordon JW. Certain molar movements of the human body produced by the circulation of the blood. Journal of Anatomy and Physiology 1877;11:533-536.

[5] Alexandre Laurin Andrew Blaber KT. Seismocardiograms return valid heart rate variability indices. In Computing in Cardiology. September 2013; 413-416.

[6] Jafari Tadi M, Lehtonen E, Koivisto T, Pänkäälä M, Paasio A, Teräs M. Seismocardiography: Toward heart rate variability (hrv) estimation. In Medical Measurements and Applications (MeMeA), 2015 IEEE International Symposium on. May 2015; 261-266.

[7] Ekman P, Levenson RW, Friesen WV. Autonomic nervous system activity distinguishes among emotions. Science 1983;221(4616):1208 - 1210.

[8] Electrophysiology, Task Force of the European Society of Cardiology the North American Society of Pacing. Heart rate variability: Standards of measurement, physiological interpretation, and clinical use. Circulation 1996; 93(5):1043-1065.

[9] Jia W, Li Y, Bai Y, Mao ZH, Sun M, Zhao Q. Estimation of heart rate from a chest-worn inertial measurement unit. In 2015 International symposium on bioelectrics and bioinformatics (ISBB). IEEE, 2015; 148-151.

[10] Hernandez J, McDuff D, Picard R. Bioinsights: Extracting personal data from still wearable motion sensors. In Wearable and Implantable Body Sensor Networks (BSN), 2015 IEEE 12th International Conference on. June 2015; 1-6.

[11] Nakano M, Konishi T, Izumi S, Kawaguchi H, Yoshimoto M. Instantaneous heart rate detection using short-time autocorrelation for wearable healthcare systems. In Engineering in Medicine and Biology Society (EMBC), 2012 Annual International Conference of the IEEE. ISSN 1094-687X, 2012; 6703-6706.

[12] Bruser C, Winter S, Leonhardt S. How speech processing can help with beat-to-beat heart rate estimation in ballistocardiograms. In 2013 IEEE International Symposium on Medical Measurements and Applications (MeMeA). IEEE, 2013; 12-16.

[13] Shimamura T, Kobayashi H. Weighted autocorrelation for pitch extraction of noisy speech. IEEE Transactions on Speech and Audio Processing 2001;9(7):727 - 730. ISSN 1063-6676.

[14] Tarvainen MP, Niskanen JP, Lipponen JA, Ranta-aho PO, Karjalainen PA. Kubios HRV heart rate variability analysis software. Computer Methods and Programs in Biomedicine 2014;113(1):210 - 220. ISSN 0169-2607.

[15] Pan J, Tompkins WJ. A Real-Time QRS Detection Algorithm. Biomedical Engineering IEEE Transactions on March 1985;BME-32(3):230-236.

Address for correspondence:

Olli Lahdenoja

Technology Research Center, University of Turku olanla@utu.fi 